\title{
Un exemple des limites du calque latin dans la description du nom : la théorie des cas au XVIIIle siècle
}

\author{
An example of the limits of the Latin calque in the description of \\ the name: the case theory in the $18^{\text {th }}$ century
}

Sévrine Dagnet

Université Sorbonne Nouvelle - Paris 3

\begin{abstract}
Résumé : Au moment de décrire les parties d'oraison du français, les grammairiens des XVIle et XVIIIe siècles se tournent, comme leurs prédécesseurs, vers le latin, perçu comme un modèle d'excellence. Plus spécifiquement en ce qui concerne le nom, cette imitation les conduit à décréter que cette occurrence varie comme son homologue antique en genre, en nombre et en cas. La difficulté liée à l'absence de morphème casuel du substantif français, loin de constituer un frein, est contournée: en français la déclinaison du nom serait surtout celle de l'article, occurrence antéposée qui tout en précisant la référence opérée par l'élément nominal, varie au gré de la fonction grammaticale occupée par le groupe nominal. Ainsi, l'occurrence la femme (sujet ou complément d'objet) correspondrait au nominatif et à l'accusatif, tandis que l'occurrence à la femme (complément d'attribution) équivaudrait à un datif. Cette théorie, déjà en péril aux $X V \mid e$ et $X V \|$ e siècles, est très largement remise en cause au XVIIIe siècle. La controverse ne décourage pas Régnier-Desmarais (1706) et Vallart (1744), qui défendent la thèse d'une variation en cas et établissent des paradigmes de déclinaisons, longuement explicités et justifiés (première partie). Leurs argumentations étayées se heurtent aux exposés de Girard (1744) et de Du Marsais (1769), qui perçoivent le calque du latin comme un contresens syntaxique et une méconnaissance des caractéristiques de l'article, classe de mots en mal de statut (deuxième partie). Beauzée (1767) réconcilie les deux tendances en affirmant que si le nom ne possède pas de déclinaison, le français reste une langue casuelle en ce qui concerne le pronom. Rétablissant ainsi la légitimité du cas tout en le limitant à un type de mots précis, il s'interroge plus globalement sur le rôle de cette catégorie grammaticale (troisième partie).
\end{abstract}

Mots-clés : nom, article, parties d'oraison, grammaires françaises du XVIIe siècle, grammaires françaises du XVIIII siècle.

Abstract: When describing the different components of French speech, the grammarians of the sixteenth and seventeenth centuries resorted, like their predecessors, to Latin, viewed then as a model of excellence. More specifically, as far as the noun is concerned, their emulation led them to declare that its occurrence varies in gender, number, and case, just as with its ancient counterpart. Far from constituting an obstacle, the difficulty surrounding the lack of case morphemes in the French substantive is circumvented: in French, the declension of the noun is more properly that of the article, an anteposed occurrence that both clarifies the reference implied by the noun element and varies according to the grammatical function occupied by the noun group. Thus the occurrence la femme (subject or direct object) corresponds to the nominative and the accusative, while the occurrence à la femme (indirect object) would equate to a dative. This theory, already endangered in the sixteenth and seventeenth centuries, was very widely challenged in the eighteenth century. The controversy did nothing to discourage Régnier-Desmarais (1706) and Vallart (1744), who defend the theory of case variation and establish declension paradigms that they clarify and justify at length (first part). Their wellsupported arguments clash with reports by Girard (1744) and Du Marsais (1769), who perceive the Latin calque as a syntactic misinterpretation and a misunderstanding of the characteristics of the article, at 
the time a poorly-regarded class of words (second part). Beauzée (1767) reconciles the two trends, claiming that even if the noun does not undergo declension, French remains a case language as far as the pronoun is concerned. Thereby restoring the legitimacy of the case while limiting it to a precise type of word, he more generally questions the role of this grammatical category (third part).

Keywords: noun, article, parts of speech, $17^{\text {th }}$-century French grammars, $18^{\text {th }}$-century French grammars.

\section{Introduction : Contexte théorique et problématiques}

Au moment de décrire les différentes classes de mots du français, les grammairiens des XVIIle et XVIIIle siècles se tournent, à l'instar de leurs prédécesseurs, vers le modèle latin, qui représente le seul canevas théorique connu et maîtrisé. Le calque latin sur le français, comme tout "transfert technologique "', réclame des ajustements, des adaptations, mais induit aussi des remises en cause. En effet, entre les partes orationis latines et les parties d'oraison françaises, la symétrie est imparfaite. L'observation du traitement du nom dans les grammaires françaises des XVIIe et XVIIIe siècles en est un exemple remarquable.

Outre sa capacité à former un énoncé complet aux côtés du verbe, le nom est, pour reprendre la terminologie en vigueur dans les ouvrages des XVIIe et XVIIIe siècles, "la partie déclinable $\|^{2}$ susceptible de recevoir le plus grand nombre de variations, parmi lesquelles le genre, le nombre, le $\mathrm{cas}^{3}$. Le dernier, évoqué pour la première fois par Aristote, aussi bien au sujet du nom que du verbe ${ }^{4}$, dans la Poétique et dans De l'interprétation, est incontestablement celle qui fait le plus débat. En effet, la question de la théorie casuelle s'inscrit dans le prolongement d'une réflexion déjà ancienne, laissée en héritage. Rappelons en effeł qu'au XVle siècle, le modèle casuel du latin largement plébiscité ${ }^{5}$ ne fait pourtant pas l'unanimitéb.

De même, dans le corpus, deux thèses s'affrontent. Les défenseurs des cas, davantage orientés vers une approche morphologique des classes de mots, perçoivent le calque comme une évidence morphosyntaxique et comme la preuve d'une filiation illustre entre le français et le modèle antique (Irson (1656), Chiflet (1659), Régnier-Desmarais (1709), Vallange (1723), Vallart (1744), l'abbé d'Olivet (1767, 1771)). Au contraire, les opposants l'analysent comme une erreur d'interprétation (Port-Royal (1660) et Du Marsais (1769)) et même pour certains comme un refus d'analyser le français comme une langue autonome, libérée du joug latin (Girard, 1747). Tout l'intérêt de l'analyse réside dans la mise en lumière des

\footnotetext{
1 Nous empruntons l'expression à Sylvain Auroux (1989) qui désigne ainsi l'adoption des classes de mots grecques par les Latins.

2 Dans une dimension morphologique, certains grammairiens du corpus proposent de diviser les classes de mots en deux ensembles: les parties déclinables subissent des variations (nom, verbe, pronom, participe) contrairement aux parties indéclinables (adverbe, préposition, conjonction).

3 Ces variations morphologiques sont traditionnellement appelées par les auteurs du corpus « accidents du nom $)$.

${ }^{4}$ Selon J. Lallot (2012), ce sont les stoïciens qui sont à l'origine de la restriction des cas au seul domaine nominal.

5 Entre autres par Meunier (Grammaire françoise, 1557), R. Estienne (Traicté de la grammaire françoise, 1557), Pillot (Institution de la langue françoise, 1561), ou encore Bosquet (Élémens ou institutions de la langue françoise, 1586).

${ }^{6}$ L. Meigret (Tretté de la la grammere Francoeze, 1550).
} 
différents arguments théoriques qui soutiennent ces deux positions contradictoires et leurs répercussions sur la théorisation de l'article, questionnement complexe intimement lié à la problématique des cas.

La lecture assidue du corpus apporte en effet des réponses à une série de questions qui tiennent une place importante dans l'histoire des théories linguistiques. D'une part, comment la variation casuelle des noms est-elle justifiée, à une époque où sa validité est remise en cause par la majorité des auteurs? Dans cette même approche, comment sont décrits les différents cas du français? Comment les absences d'adéquation entre le français et le latin sont-elles traitées ? D'autre part, quelles sont les différentes attaques théoriques proposées par les opposants au calque ? Entre ces deux extrêmes, une conciliation est-elle envisageable et si oui, sous quelle forme et pour quel enjeu?

Notre enquête s'articule autour de trois parties. La première expose les caractéristiques majeures de la théorie des cas, à travers les développements de Régnier-Desmarais (1706) et de Vallart (1744), qui dans le cadre d'une description essentiellement morphologique du nom, revendiquent et justifient le calque latin. La deuxième partie consiste en l'analyse d'arguments opposés, à travers les présentations de Girard (1744) et de Du Marsais (1769), qui réduisent la théorie casuelle à la simple variation de prépositions et introduisent ainsi une réflexion sur la détermination du nom. Enfin, à la lumière de la grammaire générale, il est question, avec Beauzée (1767), d'une réappropriation de la théorie et d'une interrogation sur la finalité de la variation, élevée au rang de catégorie générale et universelle, parallèlement au genre et au nombre. L'auteur établit un pont entre les deux approches précédemment décrites en réfutant l'hypothèse de la modification casuelle du nom. Par ailleurs, il reconnaît l'existence d'une déclinaison des pronoms.

\section{Deux exemples représentatifs de la théorie des cas dans le corpus : Régnier- Desmarais (1706) et Vallart (1744)}

Si plusieurs décennies séparent le Traité et la Grammaire françoise, certaines similitudes autorisent la comparaison. Ainsi Vallart, même s'il multiplie les références à des théoriciens qui suivent une voie bien différente, love l'érudition et le caractère précurseur de Régnier-Desmarais, dans sa «Préface »:

Nous avons déjà un grand nombre de Grammaires Françoises; mais dans ce grand nombre, il n'y en a guères que trois qui ayent mérité l'eftime du Public: ce sont celle de M. l'Abbé Regnier Detmarais [...] celle du P. R Buffier [...] \& celle de M. Restaut [...]. La Grammaire de M. Regnier eft tavante \& exacte [...] (p. III-IV).

L'hétérogénéité des influences du grammairien peut surprendre. Buffier (1709) et Restaut (1730) s'inscrivent dans la lignée de la grammaire générale, qui après PortRoyal (1660), s'intéresse aux mécanismes universels du langage. Le Traité de Régnier-Desmarais, qui prétend livrer un savoir exhaustif sur la langue, se présente comme une grammaire particulière du français dont le caractère latinisant (en particulier en ce qui concerne l'étude des classes de mots) paraît déjà désuet au moment où Vallart prend la plume. Ce dernier, à l'instar de son prédécesseur, entend cependant renover avec la présentation traditionnelle des parties d'oraison. De fait, les deux auteurs rédigent des exposés qui témoignent de la résistance de la 
théorie casuelle à une époque où elle est de plus en plus discutée7. Au-delà des points communs, la présentation qui suit fait apparaître également une divergence fondamentale entre les deux approches. Dans un premier temps, tous deux s'accordent pour noter l'existence d' " affinités 8 " entre certains cas.

Ainsi, Régnier-Desmarais évoque des similitudes sémantiques et syntaxiques entre le nominatif et le vocatif, qui se voit pour cette raison accorder la deuxième place du classement et des similitudes morphologiques entre le nominatif et l'accusatif :

[...] Tous les cas de chaque nombre, tont appellez en termes de Grammaire, nominatif, genitif, datif, accufatif, vocatif, \& ablatif. C'eft ainti que la plufpart des Grammaires anciennes \& modernes ont accouttumé de ranger \& d'appeler tous les cas; mais comme le vocatif a de grands rapports avec le nominatif, on croit ne devoir pas tuivre icy le metme ordre, pour ne pas embarraffer inutilement les idées, en teparant des chofes qui ont tant de convenance l'une avec l'autre. Car premierement, outre l'affinité qu'il y a entre nommer, qui eft la fonction du nominatif, \& appeller, qui eft celle du vocatif; le vocatif a encore cela de commun avec le nominatif, qu'il régit quelquefois le verbe, tans en eftre jamais régi ; \& qu'il te joint au verbe fubstantif eftre, ce qui ne convient à aucun des autres cas. C'eft pourquoy on donnera icy le fecond rang au vocatif [...] Au refte, l'accutatif ne differe en rien du nominatif, quant à sa forme [...] (p. 143145).

Chez Vallart, la ressemblance morphologique entre le nominatif et l'accusatif, d'une part et le génitif et l'ablatif d'autre part, s'accompagne d'une remise en cause timide du dernier; remise en cause qui témoigne du défaut d'adhérence du calque : il n'y aurait en réalité que cinq cas véritables en français :

Il y a en françois comme en latin tix cas, le nominatif, le vocatif, le génitif, le datif, l'accutatif \& l'ablatif. Le nominatif et l'accutatif tont toujours temblables, de même que le génitif \& l'ablatif. On pouroit cependant ne dittinguer en françois que cinq cas, en retranchant l'ablatif, qui dans tous les mots déclinables, tant noms que pronoms, eft toujours temblable au génitif. Ce teroit la une conformité que notre langue auroit avec la gréque. Néanmoins nous admettons dans cet ouvrage le cas ablatif pour rendre mieux raifon de certains rapports (p. 92).

Les deux grammairiens établissent une bipartition d'ordre syntaxique qui puise ses origines dans la grammaire alexandrine' : le nominatif est appelé cas direct parce qu'il sert à exprimer le sujet de la proposition et qu'il est perçu comme le cas originel.

\footnotetext{
7 Dans leur Grammaire générale, les auteurs de Port-Royal conservent les noms des différents cas latins pour décrire les fonctions syntaxiques (le nominatif pour le sujet, l'accusatif pour le complément d'objet, etc.). Mais il s'agit là d'un choix par défaut qui s'explique par l'absence d'un métalangage plus adapté. Pareillement, si les différents cas sont décrits (p. 74-81), c'est seulement parce que l'ambition des auteurs dépasse la seule analyse du français. Présents dans d'autres langues, les cas sont ainsi considérés comme une catégorie générale. Les auteurs sont en effet formels : « Il eft vrai que de toutes les Langues il n'y a peut-être que la Grecque \& la Latine qui aient proprement des cas dans les noms $»($ p. 73).

${ }^{8}$ Nous empruntons ce terme à J. Lallot (2012:135-146), qui insiste sur les ressemblances sémantiques et morphologiques de certains cas du grec, mises en lumière notamment par les grammairiens alexandrins.

9ll s'agit là d'un apport des stoïciens, qui contrairement à Aristote, choisissent de considérer le nominatif comme un cas à part entière.
} 
II se distingue en cela des autres, appelés cas obliques ou cas indirects (vocatif, génitif, accusatif, datif, ablatif) qui s'inscrivent dans un rapport de dépendance syntaxique beaucoup plus marqué10.

L'explication étymologique de chaque cas et l'énumération des propriétés qui y sont attachées, viennent par la suite conforter l'idée d'une volonté de coller au plus près du latin.

Pour le nominatif, si Vallart se contente d'une définition purement sémantique"l Régnier-Desmarais insiste davantage sur le rôle syntaxique du cas :

Le propre du nominatif eft de regir tousjours le verbe : \& toutes les fois que la chofe ou la perfonne qu'on nomme, regit le verbe, comme le ciel eft ferein, la terre rapporte tous les ans, Alexandre vainquit Darius, il eft conttant qu'alors le nom dont on te tert pour exprimer la chote ou la perfonne ne peut eftre qu'au nominatif (p. 145).

Tout comme le rapprochement possible entre le génitif et l'ablatif souligné par Vallart, le calque du vocatif, qui se passe volontiers de l'article, fragilise la thèse avancée par les auteurs. En effet, si c'est bien cette occurrence qui porte en français la marque de la flexion, on comprend difficilement comment on peut admettre l'existence d'un cas qui se caractérise par une absence de détermination. L'évocation du remplacement de l'article par la "particule» $\hat{0}$, comme dans l'exemple Ô Seigneur, exaucez-nous (Régnier-Desmarais, 1706 : 144) peine à convaincre, notamment à cause de son caractère facultatif. À cela s'ajoute le fait que le vocatif bénéficie d'un statut mixte à la frontière du nominatif12:

Le vocatif que nous regardons icy comme le tecond cas, eft ainti nommé, parce que c'ett de celuy-là qu'on te tert, quand on appelle quelqu'un, ou qu'on luy addreffe la parole. II manque abtolument d'article, mais on y tupplée par la particule 0 , de mefme qu'en plutieurs autres Langues. Du refte, il a comme le nominatif le privilege de regir le verbe, tans en eftre jamais regi: mais il ne le regit que quand le verbe eft mis à l'imperatif, Seigneur exaucez-moi; ou quand on l'employe, foit par exclamation, toit par interrogation, ô juste ciel souffrirez-vous ! [...]. Ce qu'il a de particulier, c'eft qu'on le peut employer tolitairement tans le joindre à aucun verbe qu'il regiffe Seigneur je n'ay d'esperance qu'en vous [...]. E† en cela il eft different du nominatif qui regit toufours un verbe, ou exprimé, ou foufentendu (Régnier-Desmarais, 1706 : 143-144).

Le génitif, qui se caractérise par une pluralité de relations (causalité, partition, appartenance, etc.), donne lieu à un développement beaucoup plus fourni chez ce même auteur :

\footnotetext{
10 Dans la grammaire alexandrine (Denys le Thrace, Apollonius Dyscole, Diogène Laërce), l'opposition est déjà d'ordre syntaxique: « [...] le cas direct, comme premier actant coréférent avec la désinence verbale, s'oppose à l'ensemble formé par les cas obliques, tous susceptibles, selon la rection des verbes, d'occuper une position de deuxième actant, terme de la transitivité verbale [...] ") (J. Lallot, 2012 : 137).

11 « Le nominatif est celui dont on se sert pour nommer » (Vallart, 1744 : 93).

12 Le vocatif est déjà présenté par la grammaire alexandrine comme un cas en marge des autres de par sa capacité à exprimer un énoncé complet sans le recours au verbe (J. Lallot, 2012 : 127, 137).
} 
Le cas qu'on appelle genitif, c'eft-à-dire, qui engendre, qui produit, devroit peut-eftre avoir plutoft l'appellation paffive d'engendré, de produit. Quoy qu'il en toit, il ne s'employe jamais dans notre Langue, tans eftre gouverné par un autre nom auquel il te rapporte, toit comme l'effet à ta caute, le Createur du ciel \& de la terre, l'auteur du livre [...], toit comme la caute à son effet, l'ouvrage du Createur, le fils du Prince; toit comme le tout à ta partie, un membre du corps, [...] un arbre de la foreft; toit comme une chote potfedée à celuy qui la potfede, le Maiftre du monde, le Seigneur du lieu; toit comme le poffeffeur à la chofe potfedée, le Palais du Roy, les biens de l'Eglife ; toit comme le tujet à ton attribut, la bonté du Createur, l'ingratitude de la créature [...]; toit enfin de quelque manière que ce puiffe eftre ; les Jentiments de l'humanité, l'amour de la vie, la crainte de la mort [...] (Régnier-Desmarais, $1706: 144)$.

De même, lorsque vient le tour du datif, Régnier-Desmarais (1706 : 145) insiste sur la variété de constructions dont ce cas est susceptible. Le datif peut en effet être régi par un verbe (Plaire aux gens de bien), un participe actif (comprenons participe présent) (Servant à la messe), un participe passif (comprenons participe passé) (Occupé à l'étude), ou encore un nom substantif (Son inclinaison à la vertu), etc.

L'accusatif, qui «fait connaître la personne ou la chose qui est le terme d'une action ॥ (Vallart, 1744 : 94), ne donne pas lieu à des commentaires particuliers.

L'évocation de l'ablatif offre à Régnier-Desmarais (1706: 146) l'occasion de le distinguer du génitif avec lequel il se confond morphologiquement, à partir de traits distinctifs sémantiques (le génitif exprime généralement la relation et l'union ; l'ablatif la séparation et la division) et syntaxiques (le génitif ne peut être régi que par un nom ; l'ablatif suit un verbe ou un participe).

Cependant, si les deux grammairiens s'accordent sur l'existence des cas et des propriétés sémantico-syntaxiques de chacun, ils s'opposent néanmoins sur le nombre de déclinaisons. Régnier-Desmarais n'en admet qu'une seule. Prenant ses distances avec l'héritage théorique qu'il prônait dans sa "Préface ", Vallart reproche explicitement à son prédécesseur (ainsi qu'à Buffier (1709) qui suit le même raisonnement) d'avoir calqué aveuglément le latin, sans tenir compte des spécificités de la langue française:

Montieur l'abé Regnier \& le P. Buffier, les deux plus habiles grammairiens françois que nous aiions, ont tous deux avancé comme la chote la plus certaine du monde, que nous n'avons en françois qu'une teule déclinaiton [...]. Mais ces Meffieurs n'ont point envifagé la chofe dans ton véritable point de vue, \& leur raifonnement qui auroit été très-juste en latin, te trouve très faux en françois. En effet ce qui en latin diftingue dans un nom les cas les uns des autres, c'eft la différence des terminaitons, comme on le voit dans Dominus, domini, domino, dominum, domine, dans Pater, patris, patri, patrem, patre, \&c. Au lieu qu'en françois ce qui dittingue les cas les uns des autres, ce n'eft pas la terminaifon qui eft la même dans tout un nombre, mais ce font ou les articles ou les prépotitions de \& à qui te mettent avant les noms [...] (Vallart, $1744: 101-102)^{13}$.

\footnotetext{
13 Le reproche est d'ailleurs annoncé dès la "Préface», puisqu'au sujet des trois grammairiens qu'il a précédemment loués (pour rappel Régnier-Desmarais, Buffier et Restaut), Vallart s'autorise la critique
} 
L'auteur du Traité propose en effeł une déclinaison, présentée sous la forme de quatre paradigmes, qui prennent en compte le genre de l'article et la nature (consonne ou voyelle) de la lettre initiale du nom ${ }^{14}$. La critique formulée par Vallart, qui préfère l'adaptation, au calque irréfléchi, est assez évidente : Régnier-Desmarais ne tient pas compte du fait que si les cas français sont morphologiquement différents des cas latins ${ }^{15}$, la déclinaison doit l'être aussi. Vallart choisit une voie différente, en affirmant qu'il y a autant de déclinaisons qu'il y a de variations possibles de l'article:

Il en eft à peu près de même pour les déclinaitons. Ce qui en latin établit la différence des déclinaitons ce tont les différentes terminaitons pour le même cas ; par exemple pour le génitif tingulier : $œ$, i, is, ûs, ei : pour le datif singulier: $œ, o, i, v i$, ei; de torte qu'il y a en cette langue autant de déclinaitons qu'il y a de différentes terminaitons pour le même cas. C'eft tout le contraire en françois: ce qui établit en cette langue la différence des déclinaitons ce tont les différentes combinaitons que l'on fait des articles ou des prépotitions de \& à, pour en former le même cas; de torte qu'il y a autant de déclinaitons qu'il y a de ces différentes combinaitons (Vallart, 1744 : 102).

En conséquence, l'auteur de la Grammaire reconnaît l'existence de cina déclinaisons présentées de la page 102 à la page 11616. II est vrai que Vallart, contrairement à son prédécesseur, reconnaît les occurrences un et du comme des articles à part entière et que, de ce fait, dans son approche, ces dernières sont susceptibles comme les formes le, la, les d'une déclinaison. Voilà qui prouve une fois de plus le lien étroit entre la théorie casuelle et le corpus de l'article dans la tradition grammaticale du XVIIIe siècle.

Ainsi, les exposés de Régnier-Desmarais et de Vallart prouvent à la fois la résistance et la fragilité de la théorie casuelle. La confusion possible entre le génitif et

suivante: «lls n'ont point connu le nombre de nos déclinaitons, puifqu'ils difent que nous n'en avons qu'une teule, tandis que nous en avons cinq comme j'ai prouvé aux pages 100, $101 \& 102$ » (p. V).

${ }^{14}$ Déclinaison de l'article masculin le avant les consonnes: (singulier) Nominatif : le ciel, Vocatif : ô ciel ou ciel, Génitif : du ciel, Datif : au ciel, Accusatif : le ciel, Ablatif : du ciel - (pluriel) Nominatif : les cieux, Vocatif : ô cieux ou cieux, Génitif : des cieux, Datif : aux cieux, Accusatif : les cieux, Ablatif : des cieux (p. 147)

Déclinaison de l'article masculin le avant les voyelles : (singulier) N. : l'esprit, V. : ô esprit ou esprit, G. : de l'esprit, D. : à l'esprit, A. : l'esprit, A. : de l'esprit - (pluriel) N. : les esprits, V. : ô esprits ou esprits, G. : des esprits, D. : aux esprits, A. : les esprits, A. : des esprits (p. 148).

Déclinaison de l'article féminin la avant les consonnes: (singulier) N.: la femme, V.: ô femme ou femme, G. : de la femme, D. : à la femme, A. : la femme, A. : de la femme - (pluriel) N. : les femmes, V. : ô femmes ou femmes, G. : des femmes, D. : aux femmes, A. : les femmes, A. : des femmes (p. 148).

Déclinaison de l'article féminin la avant les voyelles: (Singulier) : N. : l'âme, V. : ô âme ou âme, G. : de l'âme, D.: de l'âme, A. : l'âme, A.: de l'âme - (Pluriel) N.: les âmes, V.: ô âmes ou âmes, G.: des âmes, D. : aux âmes, A. : les âmes, A. : des âmes (p. 149).

15 En latin, les morphèmes finals des noms varient en fonction des cas. C'est d'ailleurs l'un des principaux arguments avancés par les opposants à la thèse de la déclinaison des noms français.

16 «La première déclinaiton joint l'article défini le, la avec les noms [...]" (p. 103); "la teconde déclinaifon joint l'article indéfini un, une avec les noms [...] ॥ (p. 108) ; "la troitième déclinaiton joint l'article partitif $d u$, de la, avec les noms [...] » (p. 110) ; "la quatrième déclinaiton prend la prépotition de à trois cas qui tont le nominatif, l'accutatif \& le génitif, \& à de au datif, \& cela tant au tingulier qu'au pluriel [...]॥ (p. 112); [la] cinquième déclinaifon prend de au génitif \& à l'ablatif, \& à au datif. [...] Comme cette déclinaiton comprend les noms propre d'hommes \& de villes, elle n'a point de pluriel ॥ (p. 113). 
l'ablatif, la construction du vocatif et le débat sur le nombre de déclinaisons démontrent qu'en matière de cas, le calque du latin sied mal au français. II ne faudrait pas oublier cependant que la question des cas aux XVIIe et XVIIIe siècles est à rattacher à une problématique plus large et plus complexe, qui embarrasse l'ensemble des grammairiens du corpus. La question de l'article (et plus précisément du corpus qu'il regroupe), qui est déjà une des origines du débat sur le nombre de déclinaisons, est également au cœur de la remise en cause de la variation en cas, portée essentiellement par Girard (1747) et Du Marsais (1769), qui livrent les argumentations les plus développées.

\section{La critique des cas au service d'une argumentation en faveur de l'article chez Girard (1747) et Du Marsais (1769)}

Le rapprochement entre les Vrais principes de la langue françoise de Girard et Logique et principes de grammaire de Du Marsais demande à être explicité. Les deux ouvrages s'opposent en effet de prime abord par leur finalité. Celui de Girard se présente dès sa "Préface" comme une grammaire spécifique du français, qui entend réformer entièrement l'étude de langue en s'affranchissant de la tradition grammaticale. Cette autonomie ouvertement revendiquée prend la forme d'une mise à distance de l'héritage des grammairiens antérieurs mais surtout de l'enseignement du latin. L'oeuvre de Du Marsais se présente du propre aveu de l'éditeur, comme une compilation de textes en grande partie posthumes. S'inscrivant dans le cadre de la grammaire générale, l'ouvrage part du principe, au même titre que Port-Royal, que l'acte de parole émane de la pensée et entend identifier et décrire les mécanismes qui soutiennent le système du langage. II n'existe donc aucune influence explicite ou sous-entendue entre les deux auteurs. Cependant, malgré une divergence d'objectifs et de contenus, tous deux concourent à la critique de la théorie casuelle au XVIIIe siècle.

Ainsi, chez Girard et Du Marsais, cette dernière s'insère dans des développements qui visent la remise en cause de l'article indéfini sous les formes à de/aux/à des, attestée chez la majorité des auteurs. Le débat prend pour cadre général le questionnement sur la légitimité et sur le corpus de l'article, occurrence qui au regard du modèle latin, trouve difficilement sa place au sein des parties d'oraison ${ }^{17}$. Si les auteurs poursuivent des finalités différentes qu'il s'agira de définir, tous deux défendent néanmoins des points de vue assez proches en la matière.

Chez Girard, I'article, évoqué dans le discours IV du premier volume, est la première des dix parties d'oraison attestées. Dès le début de son analyse, à la page 153, l'auteur s'inscrit explicitement dans une volonté de rupture, là où la majorité de ses contemporains se contente d'une définition morphologique ou syntaxique ${ }^{18}$ :

[...] L'article eft un mot établi pour annoncer et particulariser timplement la chote tans la nommer; c'eft-à-dire qu'il eft une expreftion indéfinie quoique potitive, dont la jutte valeur n'ett que de faire naître l'idée d'une

\footnotetext{
${ }^{17} \mathrm{En}$ latin, l'article n'existe pas. Certains grammairiens décident d'inclure l'article dans une autre classe de mots déjà existante ((Beauzée (1767), Du Marsais (1769), l'abbé d'Olivet (1771)). Vallange (1723) décide même de proscrire l'appellation d'article au profit de celle de « monosyllabe ".

${ }_{18}^{18}$ 'article est traditionnellement présenté comme une occurrence qui précède le nom et subit les mêmes variations.
} 
efpece fubtiftante qu'on diftingue de la totalité des êtres, pour être entuite nommée (Girard, 1747, vol. 1 : 157-158).

Ainsi, ce discours est implicitement divisé en quatre axes, à savoir la définition de l'occurrence, la remise en cause de la théorie casuelle, la réfutation des formes un/une en tant qu'article (en réponse aux auteurs de Port-Royal) et enfin l'évocation des emplois de l'article. C'est essentiellement la deuxième partie qui nous intéresse ici. Après avoir critiqué les définitions de Richelet (1680) ${ }^{19}$ et de Buffier (1709) ${ }^{20}$, (p. 154-155), Girard introduit sa remise en cause des cas par un exemple qui démontre que les articles le, la et les (seules formes reconnues par l'auteur) restent invariablement les mêmes, quelle que soit la fonction syntaxique occupée par le groupe nominal. Évoquer une quelconque théorie casuelle serait donc une aberration :

Les cieux envoient vers la terre les influences qui la rendent fertile; et la terre renvoie vers le ciel les vapeurs et les exhalaijons dont fe forment les pluies et le tonnerre dans la moyenne région de l'air. II eft aisé de voir, par cette phrafe, que l'article n'admet aucune divertité d'inflexion. C'eft toujours le, la, les; quoique les dénominations qu'il annonce tantôt y régiffent le verbe, et tantôt y toient elles-mêmes régies ou par le verbe ou par des prépotitions. Commençons donc dès à prétent à purger la méthode françoite de tout ce fatras de cas, comme de tuperfluités étrangères plus propres à nuire qu'à tervir l'intelligence de notre langue (Girard, 1747, vol. $1:$ 166).

Pour son second argument, Girard s'appuie sur le phénomène de syncope, définie comme "un état mixte et composé " (p. 167) qui consiste en une contraction de l'article défini dès lors qu'il est postposé à des prépositions comme au, aux, du, des. Cette évocation n'est pas anodine: la transformation morphologique qui en résulte peut laisser croire à une variation casuelle de l'article. Girard s'emploie à démontrer qu'il n'en est rien. Pour ce faire, il note que les groupes nominaux syncopés ont leurs équivalents. C'est ainsi que les occurrences Maître du monde, Plaire au peuple sont analysées respectivement comme: Maître de tout le monde et Plaire à tout le peuple, à la page 170. La remise en question des cas est l'occasion pour le grammairien de reprendre une polémique qui lui est chère et qui apparaît périodiquement dans sa grammaire. Selon lui, l'hypothèse de la variation du nom et de l'article est à mettre au compte d'un calque systématique et abusif de la langue latine et du refus d'étudier la langue française pour et par elle-même :

Toutes ces idées de cas ne tont que des livrées de latinité, que nos grammairiens français n'ont ofé quitter par je ne tais quel efprit tervile [...].

\footnotetext{
${ }^{19}$ « La Grammaire qui annonce dans fon titre un plan nouveau, après avoir promis merveille dans fa préface, dit timplement tur ce fujet, que quelques particules Jont apelées Articles parce qu'ils Jervent à articuler \& à diftinguer certains emplois que l'ufage fait des Noms ; Elle laiffe entuite à son lecteur le toin \& la gloire de chercher lui même ce qu'elle entend par articuler [...]. De torte que la définition n'eft pas plus claire que le nom de la chote [...] ). (p. 154).

${ }^{20}$ «Le Dictionnaire de François de Richelet définit l'article comme « une petite particule qu'on met devant les Subftantifs, \& qui fert à en faire connoître le nombre, le genre \& le cas. Cette définition eft mieux faite que la précédente car aumoins elle dit quelque chote; mais par malheur elle peche dans le vrai $»($ p. 154).
} 
En étudiant au collège, on a vu que les mots latins variaient leur terminaiton par rapport aux divertités du régime dans lequel on les plaçait: que ces diverfes terminaifons étaient nommées cas et que comme dans ces écoles on n'eft occupé que de langue latine, les régents s'appliquant uniquement à la rendre intelligible à la jeuneffe par des traductions convenables, tans tonger à former des principes sur celle qu'ils parlent naturellement; il eft arrivé qu'ils ont également nommé génitif, datif en français ce qui répondait à ces cas latins dans leurs traductions tans faire attention que notre langue marque par des prépotitions le régime que la latine marque par des cas (Girard, vol. 1: 174).

L'auteur présente par la suite trois exemples de constructions pour lesquels la théorie casuelle introduit une mauvaise interprétation syntaxique. Analysons le premier proposé. Dans l'énoncé De très habiles gens tont quelque fois dupés par des tots (p. 178), le mot de laisse deviner un génitif, or le groupe nominal de très habiles gens est incontestablement le sujet de la phrase.

Dans l'exemple $n^{\circ} 3, I I$ ne s'amuse pas à de la crème fouettée quand il peut s'attacher à de la viande (p. 183-184), la cible de Girard est double, puisqu'elle vise aussi bien la critique des cas que celle des formes à de/à des, présentées comme des articles par une grande partie des grammairiens du corpus :

Il arrive aussi dans ce tystème une chofe tout à fait divertiffante. C'eft que les différents articles te raffemblent et font, pour ainfi dire, efcadronner tous leurs cas à la tête d'un feul et même nom. [...] Elles [= les dénominations crème et viande] en ont trois de front, définis et indéfinis [= des articles]. Si chacun d'eux fait fon devoir pour la fixation du cas; elles feront tout à la fois au datif, au génitif et au nominatif : ou f'ils concourent tous à marquer un feul et même cas par la force d'une union imaginée pour former un troifieme article "composé mitoyen" et bizarre, ainti que le nomme fort ingénieusement l'auteur qui l'a forgé comment et pourquoi ces articles perdent-ils là par leur proximité leur valeur essentielle ? Cela ett-il pottible ? Que de confution et de galimatias dans ces fortes de grammaires! Ett-ce ainti qu'on établit les principes et qu'on explique les loix de l'usage ? » (Girard, 1747, vol. 1 : 183-184)

Ainsi, ce prétendu article indéfini est jugé en grande partie responsable du maintien de la théorie des cas. Pour l'auteur, loin de constituer des articles ou des marques casuelles, ces occurrences sont en réalité des prépositions qui apparaissent antéposées aux articles définis, en vertu des lois syntaxiques du français. II s'en explique en ces termes:

Ces trois mots à de la, mis devant les substantifs crème et viande font de trois efpèces différentes, ayant chacun leur tens particulier et un différent emploi modificatif. Que la qui précède immédiatement le nom, eft l'article qui l'annonce [...] privé de cas comme lui. Que de, placé avant la, eft une particule qui réduit en extrait la totalité de la chofe dénommée par les fubttantifs. Qu'enfin à eft une prépotition qui a fous fon régime avec tout ce qui en compote le cortège, foit pour l'annoncer, ou pour reftreindre l'étendue du tens, ou pour le qualifier; et qu'elle fert à indiquer le rapport qu'il y a entre l'action exprimée par le verbe et le terme de cette action marqué par ces mots de la crème fovettée ou par ceux-ci de la viande (lbid. : 189). 
Dans le deuxième volume des Principes de grammaire de Du Marsais, le chapitre consacré à l'article se divise en trois parties. Dans un premier temps, après avoir présenté à la page 425 l'occurrence comme un mot sémantiquement vide qui parfait la référence opérée par le nom, l'auteur répond explicitement à RégnierDesmarais, en remettant en cause, à la suite de Girard, la théorie des cas ${ }^{21}$. Enfin, il cherche à justifier l'inclusion des articles dans la classe des adjectifs. Comme pour l'auteur des Vrais Principes, c'est surtout le développement sur la remise en cause du calque latin qui retient notre attention. Aux appellations " articles définis » et " articles indéfinis ", Du Marsais préfère, dans une dimension lexicologique, parler à la page 430 d' "articles simples » (le, la, les) et d' "articles composés » (au, aux, du, des), nés de l'association des occurrences à ou de avec les premiers. À l'instar de Girard, le grammairien s'appuie sur ces derniers, considérés comme des formes amalgamées pour remettre en question l'existence des cas. Par la suite, il s'efforce de démontrer, à l'aide de nombreux exemples, que les prépositions à et de servent à exprimer différents rapports entre les mots, là où le latin se servait de la modification morphologique des noms :

Cependant, communément nos Grammairiens ne regardent ces deux mots [de \& à] que comme des particules, qui tervent, difent-ils, à décliner nos noms. L'un [de] eft, dit-on, la marque du génitif, et l'autre [à], celle du datif. Mais, n'eft-il pas plus timple, et plus analogue au procédé des langues, dont les noms ne changent point leur dernière syllabe, de n'y admettre ni cas, ni déclinaisons, et d'observer seulement comment ces langues énoncent les mêmes vues de l'esprit, que les latins font connoître par la différence de terminaisons? Tout cela se fait ou par la place du mot, ou par le fecours des prépositions (vol. 2 : 439-440).

Mais, chez cet auteur, la critique du modèle latin est plus modérée que chez Girard. En établissant un parallèle syntaxique, Du Marsais renove avec la tradition grammaticale. Si le français ne connaît pas de variation casuelle, il adopte néanmoins, en ce qui concerne les prépositions, le même usage que le latin :

\begin{abstract}
Les Latins n'ont que tix cas; cependant il y a bien plus de raports à marquer; ce plus, ils l'énoncent par le tecours de leurs prépotitions. Hé bien, quand la place du mot ne peut pas nous fervir à faire connoître le raport que nous avons à marquer, nous faitons alors ce que les Latins faifoient au défaut de détinence ou terminaiton particulière. Comme nous n'avons point de terminaiton dettinée à marquer le génitif, nous avons recours à une prépotition. II en eft de même pour le raport d'attribution: nous le marquons par la prépotition à ou par la prépotition pour, \& même par quelques autres, \& les Latins marquoient ce raport par une terminaison particulière, qui faitoit dire que le mot étoit alors au datif (vol. 2 : 439-440).
\end{abstract}

Selon lui, en français, les prépositions ont pour rôle de remédier à l'absence de changement morphologique des noms. Cette position lui fournit un nouvel argument contre la théorie casuelle. Les prépositions françaises à et de sont héritées respectivement des formes latines ad/ab et de. Si le latin les regarde comme des

\footnotetext{
${ }^{21}$ « Les grammairiens ont apellé articles, certains mots qui ne tignifient rien de phyfique, qui tont identifiés avec ceux devant lefquels on les place, \& les font prendre dans une acception particulière. Par exemple, le roi aime le peuple; le premier le ne prétente qu'une même idée avec le peuple, mais il m'indique un roi particulier que les circonstances du pays où je tuis, ou du pays dont on parle me font entendre [...] ॥ (Girard, $1747: 425-426)$.
} 
prépositions, pourquoi le français les envisagerait-il autrement? Si l'argument peut paraître convaincant, l'analogie, poussée à l'extrême, peut à son tour faire obstacle à la thèse du grammairien. En effet, si en latin l'existence de prépositions n'empêche pas celle des cas, pourquoi en serait-il autrement en français ? Ainsi, démontrer que à et de sont des prépositions ne permet pas pour autant, du moins dans la démarche adoptée par Du Marsais, de remettre en question l'existence des cas en français. Dans un troisième temps, l'auteur évoque, comme Girard, les confusions créées par la théorie des cas, à l'aide de l'examen d'énoncés à l'interprétation problématique. Sa présentation est l'occasion d'une nouvelle critique nominative à l'encontre de l'auteur du Traité :

Tout ce que dit M. L'abbé Régnier, pour faire voir que nous avons des datifs, me paroît bien mal afforti [...] quand felon ce grammairien, quand on dit, Voilà un chien qui s'est donné à moi, à moi eft au datif. Mais ti l'on dit, Un chien qui s'est donné à moi, ce moi, n'eft plus alors au datif, c'eft, dit-il, la prépotition latine ad. J'avove que je ne saurois reconnoître la prépotition latine dans adonné à, tans la voir aussi dans donné à, et que dans l'une ou dans l'autre de ces deux phrases, les deux à me paraiffent de même efpèce, et avoir la même origine. En un mot, puifque ad aliquem ou $a b$ aliquo ne tont point des datifs en latin, je ne vois pas pourquoi à quelqu'un pourrait être un datif en français » (vol. 2 : p. 448-449).

En guise de transition, nous insisterons, après l'opposition implicite entre grammaire spécifique et grammaire générale, sur une autre différence fondamentale entre les deux auteurs. Tandis que Girard cherche à légitimer l'article comme partie d'oraison autonome, en s'affranchissant du joug latin, Du Marsais entend s'appuyer sur le modèle antique pour justifier l'inadéquation du calque. Cependant, tous deux dénoncent l'existence d'un article indéfini à de/à de la/aux, responsable de l'illusion casuelle et par là même de la méconnaissance de la classe.

Néanmoins, le clivage entre les adeptes des cas (Régnier-Desmarais et Vallart dans la première partie) et les opposants (Girard et Du Marsais dans la deuxième) aussi intéressant qu'il soit, enferme la réflexion dans un système binaire (les noms du français varient en cas/ces mêmes noms ne varient pas en cas) évité par Beauzée, qui s'intéresse entre autres à la finalité des cas, dans sa Grammaire générale.

\section{De la raison d'être des cas selon Beauzée (1767)}

De par le titre de sa grammaire22, Beauzée s'inscrit explicitement dans la lignée d'Arnault et de Lancelot (1660). Cet héritage théorique est réaffirmé dès la "Préface", notamment dans la distinction entre la grammaire générale et la grammaire particulière et la mention de l'assujettissement de la seconde à la première (p.X-XI). La grammaire générale élevée au rang de "science" est première par rapport à la grammaire particulière, qui n'est qu'une de ses applications individuelles (p. X) ${ }^{23}$.

\footnotetext{
${ }^{22} \mathrm{Cf}$ : Grammaire générale ou Exposition raisonnée des éléments nécessaires du langage, pour servir de fondement à l'étude de toutes les langues.

${ }^{23}$ « La GRAMMAIRE GÉNÉRALE eft donc la fcience raitonnée des principes immuables \& généraux du Langage prononcé ou écrit dans quelque langue que ce foit.

Une GRAMMAIRE PARTICULIÈRE eft l'art d'appliquer, aux principes immuables \& généraux du Langage prononcé ou écrit, les intitutions arbitraires \& ufuelles d'une langue particulière.
} 
Plutôt que de plaquer le modèle antique sur le français comme le font RégnierDesmarais et Vallart ou de le rejeter comme le fait Girard, Beauzée décide dans I'article VIII de son Livre III (vol. 2) d'étudier les manifestations casuelles pour ellesmêmes, c'est-à dire telles qu'elles se présentent dans une langue donnée. Son argumentation s'articule autour de trois axes, à savoir premièrement l'évocation des cas dans les langues vulgaires, puis la critique de l'article « Accusatif » rédigé par Du Marsais (Dictionnaire Raisonné) et enfin la question de la motivation des cas.

Le point de départ de la réflexion est le commentaire de Sanctius, qui dans sa Minerve publiée en 1587, présente l'adoption des six cas latins par les langues vulgaires comme nécessaire et naturelle:
Sanctius prétend que la divition des Cas latins en tix eft naturelle; In omni porrò nomine natura sex partes constituit : \& il en conclut qu'elle doit être la même dans toutes les langues; Quoniam haec Casuum partitio naturalis est, in omni item idiomate tot Casus reperiri fuerit necesse. C'eft fur ce principe qu'il établit entuite que les grecs ont \& doivent avoir un ablatif. [...] Cela étant, comment eft-il potfible de concilier l'affertation de Sanctius fur la néceffité univerfelle des tix Cas adoptés dans la langue latine, avec les utages combinés des autres langues? Afin d'en mieux tentir la difficulté, arrêtons-nous un moment tur les différents procédés des unes \& des autres (Livre III: 151).

Par la suite, Beauzée se démarque de ses prédécesseurs par une volonté de réappropriation personnelle et réfléchie de la théorie casuelle aux dépends d'une opération de calque automatique et immédiate. Pour ce faire, il reprend l'argument de Girard sur l'absence de variation casuelle des noms. Ainsi, selon Beauzée, en français, seuls les pronoms sont susceptibles de recevoir des cas:

Il faut obterver d'abord que plutieurs langues n'ont point admis de Cas pour les noms ni pour les adjectifs, mais que toutes en ont admis pour les pronoms. Ainti l'italien, l'espagnol, le portugais, l'anglois, le françois, \&c qui n'ont point donné de Cas à leurs noms ni à leurs adjectifs, en ont donné plus ou moins à leurs pronoms (p. 152).

Cependant, à l'intérieur des langues vulgaires, le système casuel n'est pas uniforme. L'évocation de l'anglais offre à l'auteur l'occasion d'introduire un nouveau terme qui puise sa raison d'être dans son rôle syntaxique :
[...] II y a un Cas que j'appelle complétif, tant pour la Grammaire angloise que pour la françoise. C'eft le feul que nos ufages ayent deftiné à marquer le complément de toutes les prépotitions. Les Latins en avoient dettiné deux à cette fin, \& il étoit néceffaire qu'ils euffent chacun un nom propre ; ils les nommèrent accusatif \& ablatif. Peut-être auroit-on mieux aimé que le nôtre eût pris le nom d'accutatif, n'eût-ce été que pour éviter une nouvelle dénomination. Mais j'ai craint que cette dénomination, pour être trop

\footnotetext{
La grammaire générale eft une science, parce qu'elle n'a pour objet que la fpéculation raitonnée des principes immuables \& généraux du Langage.

Une Grammaire particulière eft un art, parce qu'elle envifage l'application pratique des inftitutions arbitraires \& usuelles d'une langue particulière aux principes généraux du Langage. [...]

La justeffe \& la néceffité de cette diftinction, toute abttraite qu'elle paroît être, ont toutefois des caractères ti palpables, qu'elles ont été tenties de bonne heure par les bons efprits » (p. X-XI).
} 
connue en latin, n'enduisît en erreur, \& ne fît croire à quelques-uns que ce Cas n'a effectivement trait qu'à certaines prépofitions, comme l'accutatif latin; d'ailleurs nous avons vu que l'accutatif \& l'ablatif des latins tont effentiellement complétifs, \& le Cas dont il s'agit ici répond aux deux ; la dénomination la plus jufte qu'on pût lui donner eft donc celle même de complétif (Beauzée, 1767, Livre III : 154-155).

Présent aussi bien en anglais qu'en français, ce complétif se présente comme l'équivalent de l'accusatif et de l'ablatif, mais Beauzée refuse d'utiliser ces termes pour éviter toute confusion avec le latin, comme il s'en explique aux pages 154 et 155.

Le français témoigne, quant à lui, d'une répartition différente, puisque que les pronoms sont susceptibles de recevoir trois cas, à savoir le nominatif (ou le vocatif pour le pronom de la deuxième personne), le datif et le complétif, comme en témoigne la répartition de la page $153^{24}$.

La mise à distance du modèle antique n'empêche pas Beauzée de reconnaître certaines des similitudes sémantiques et syntaxiques entre les cas latins et les cas des langues vulgaires, plus particulièrement en ce qui concerne le nominatif, le vocatif et le datif :

Celui que je nomme nominatif ou vocatif [...] répond exactement aux Cas latins de même dénomination: \& c'eft la même chofe de celui que j'appelle datif dans les pronoms françois; c'eft un cas équivalant à une prépotition de tendance avec le pronom pour complément (Beauzée, 1767, Livre III : 153).

À présent, laissons de côté la critique de l'article "Accusatif», pour présenter la conclusion de Beauzée au sujet de la raison d'être des cas. Abandonnant le cas du français pour un point de vue plus général, le grammairien remarque que la variation en cas ne concerne que quatre éléments d'oraison ayant une caractéristique commune, à savoir le nom, le pronom, l'adjectif (qui comprend chez cet auteur, comme chez Du Marsais, l'article) et les participes. Ces quatre types d'occurrences ont en effet la propriété de "prétenter à l'efprit les idées des êtres, toit réels, toit abttraits, qui peuvent être les objets de notre pentée " (Beauzée : 172). La question de l'utilité des cas incite Beauzée à subdiviser les occurrences en deux sous-groupes: les cas des noms et des pronoms garantissent l'acte de parole, tandis que ceux des adjectifs et des participes sont contraints et régis par des règles de concordance.

Les noms, les pronoms, les adjectifs, \& ce qu'on appelle participes dans les verbes, tont généralement tutceptibles de Cas; \& cette propriété tient apparemment à la signification fondamentale commune aux quatre efpèces de mots déclinables, contifte à prétenter à l'efprit les idées des êtres, toit réels foit abttraits, qui peuvent être les objets de notre pentée ( $p$. 172).

$[\ldots]$

\footnotetext{
${ }^{24}$ ]ère personne - Pour le singulier : Nominatif je, Datif Me, Complétif Moi; Pour le pluriel : Nous à tous les

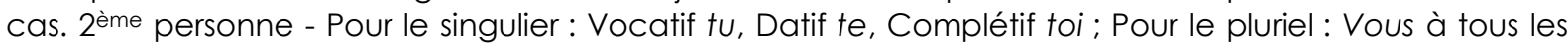
cas. 3ème personne (pronom direct) - Pour le singulier: N. il/elle, D. lui, C. lui/elle; pour le pluriel: $\mathrm{N}$. ils/elles, D. leur, C. eux/elles. 3ème personne (pronom réfléchi pour le singulier et le pluriel) : N. pas d'occurrence, D. se, C. soi.
} 
Une teconde obtervation également importante, c'eft que le choix des Cas, comme celui des nombres, te règle par deux tortes de vûes. $1^{\circ} \mathrm{Ce}$ tont les betoins de l'énonciation, d'après ce qui exitte dans l'efprit de celui qui parle, qui fixent le choix des Cas pour les noms \& les pronoms. $2^{\circ} C^{\prime}$ eft une raison d'imitation \& de concordance qui en décide pour les adjectifs \& les participes. Ainti, dans [...] Ad rivum eundem lupus \& agnus nenerant siti compulsi ; le nom rivum eft à l'accufatif, parce qu'il exprime le terme conféquent du rapport général de tendance, désigné par la préposition ad; les noms lupus \& agnus tont au nominatif, parce que chacun des deux exprime une partie du sujet total de la propotition \& du verbe venerant : voilà des raitons de néceffité, en voici d'imitation. L'adjectif eundem eft à l'accutatif, pour s'accorder en Cas avec fon corrélatif rivum : le participe compulsi est au nominatif, pour s'accorder en Cas avec les noms lupus \& agnus auxquels il eft appliqué (pp. 172-173).

\section{Conclusion: Que retenir de la théorie des cas dans les grammaires du XVIIIe siècle?}

Loin de chercher l'exhaustivité, notre analyse visait à proposer des développements représentatifs des deux grands courants d'opinion concernant la variation casuelle au XVIII siècle, problématique centrale au coeur de la théorisation du nom.

Notre première partie s'intéressait à la justification de la théorie et à l'observation de la mise en œuvre du calque latin par Régnier-Desmarais et Vallart, à une époque où l'hypothèse d'une déclinaison du nom est de plus en plus marginalisée. L'exposé a mis en avant, notamment au sujet du nombre de déclinaisons, un manque d'uniformité qui tend à affaiblir l'ensemble de la théorie.

La deuxième partie avait pour objectif d'isoler les arguments essentiellement syntaxiques avancés par Girard et Du Marsais pour réfuter la variation casuelle des noms. Elle a également permis d'isoler la cause principale de la persistance de la théorie casuelle : la méconnaissance de l'article. La difficulté liée au repérage de l'occurrence, associée à une confusion entre l'article et la préposition (les formes à de/aux et leur paradigme sont considérés généralement comme des articles définis) a fortement entretenu le mythe de la variation et de la déclinaison.

Enfin, en réfutant la variation des noms et des articles, mais en évoquant celle des pronoms, Beauzée apporte une réponse intermédiaire, tout en ajoutant au débat la question intéressante de la finalité de la modification, occultée par les autres auteurs concentrés exclusivement sur la justification ou la critique du calque. Si l'auteur de la Grammaire générale se démarque en cela de ses prédécesseurs et de ses contemporains, il n'est pas le premier à s'extraire du clivage défenseurs/opposants.

Dans la première moitié du XVIIe siècle, Maupas (1632) se distingue déjà par sa franchise et sa clarté, à une période où la théorie des cas demeure la norme. II considère en effet le français comme une langue non casuelle et le recours au calque comme une imitation sans fondement linguistique, mais nécessaire à l'enseignement. Dans la Grammaire et syntaxe, les cas sont conservés uniquement par commodité.

On tient là sans doute l'une des explications de la longévité du débat sur la théorie casuelle aux XVle, XVIle et XVIIIe siècles: faute d'un cadre formel qui leur soit propre, les classes grammaticales du français sont condamnées à être analysées à 
travers le spectre du latin, modèle d'étude à la fois familier et rassurant, quoiqu'en partie, inapproprié.

\section{Bibliographie}

AUROUX S. (1989) : Histoire des idées linguistiques, Coll. Philosophie et Langage, Pierre Mardaga, vol. 1.

BEAUZÉE N. (1767) : Grammaire générale et raisonnée ou Exposition raisonnée des éléments nécessaires du langage, pour servir de fondement à l'étude de toutes les langues; Paris, J. Barbou, 2 vol.

BUFFIER C. (1709) : Grammaire françoise sur un plan nouveau : pour en rendre les principes plus clairs et la pratique plus aisée; Paris, France-expansion, 1972.

CHESNEAU DU MARSAIS C. (1769) : Logique et principes de grammaire, Paris, Hachette, 1971, 2 vol.

CHIFLET L. (1659) : Essay d'une parfaite grammaire de la langue françoise : où le lecteur trouvera en bel ordre tout ce qui est de plus nécessaire [...], Paris, Champion, 1972.

D'OLIVET (Thoulier) P.-J. [1767] (1771) : Remarques sur la langue française, Genève, Slatkine reprints, 1968, 2e édition.

GIRARD G. (1747) : Les vrais principes de la langue françoise ou la parole réduite en méthode, conformément aux loix de l'usage, Genève, Librairie Droz, 1982, 2 vol.

IRSON C. (1656) : Nouvelle méthode pour apprendre facilement les principes et la pureté de la langue françoise, contenant plusieurs Traitez De la Prononciation, De l'Orthographe [...], Genève, Slatkine reprints, 1973.

LALLOT J. (2012) : Études sur la grammaire alexandrine, Paris, J. Vrin.

MAUPAS Ch. (1632) : Grammaire et syntaxe française contenant reigles bien exactes et certaines de la prononciation, orthographe, construction \& usage de notre langue [...], Paris, France-expansion, 1973, réimpression de la 2e édition.

RÉGNIER-DESMARAIS F. S. [1705] (1706) : Traité de la grammaire françoise, Genève, Slatkine reprints, 1973, réimpression de la $2^{e}$ édition.

RESTAUT P. (1730) : Principes généraux et raisonnés de la grammaire françoise : par demandes et réponses [...] , Paris, France-expansion, 1973.

VALLANGE (de) [1719] (1721) : Grammaire française raisonée qui ensègne la pureté et la délicatesse de la langue avec orthographe [...], Paris, France-expansion, 1973, 2e éd.

VALLART J. (1744) : Grammaire françoise, Paris, France-expansion, 1972. 